\title{
A systematic approach to constant power load stabilization by passive damping
}

\author{
J.C. Mayo-Maldonado and P. Rapisarda
}

\begin{abstract}
We illustrate a novel design methodology for the stabilization of power converters with constant-power loads by passive damping. In our formulation of the problem we allow the destabilizing loads to be modeled by by frequencydependent negative impedances. Our methodology is based on the solution of bilinear- and linear matrix inequalities, for which standard numerical solvers can be used.
\end{abstract}

\section{INTRODUCTION}

Distributed power systems consist of the interconnection of several subsystems (e.g. generators, switching power converters, loads, etc.). Each subsystem is designed separately and its stability is thus assured by design; however, their interaction with each other in the overall system can display unstable behaviors (see [2], [20], [22]). In some cases, such interactions can be accurately modelled assuming that some components behave as constant power loads, leading to negative-impedance instability [3].

Given its importance for several areas of applications, the constant power load stability problem has been studied from different points of view, see e.g. the compendium of traditional stability criteria for DC distributed power systems in [6]. In order to deal with instability, stabilizing feedback controllers for the power converters in the distribution system can be used, however such technique may be in conflict with other control objectives such as voltage/current regulation [21]. Other methods propose stabilization by passive damping (see e.g. [1]), where a passive load (filter) is interconnected to the system in order to guarantee stability. In such approach the main issue is how to design the stabilizing filter impedance, while also satisfying additional specifications, e.g. minimizing power losses.

In this paper we present a design methodology for such passive-damping filters, based on a novel approach to model linear differential switched systems, that also provides accurate piecewise linear approximations of the behavior of many power systems (see [8], [10], [11], [12]). This framework offers several advantages over the classical one based on state space descriptions sharing the same state space (see [9], [11]). Moreover, in our framework it is straightforward to accommodate more general descriptions of the instabilitygenerating mechanism than the traditional negative resistance one (see [1]), e.g. frequency-dependent negative impedances. Our methodology is based on the solution of bilinear- and

Vision, Learning and Control Group, School of Electronics and Computer Science, University of Southampton, SO17 1BJ Southampton, UK pr3eecs.soton.ac.uk. linear matrix inequalities (B/LMIs in the following), for which efficient numerical solvers exist (see for instance [7]).

This paper is organized as follows. In section II we illustrate the essential concepts of the linear switched differential systems approach and of quadratic differential forms. In section III we state formally the constant power load problem in our setting. In section IV we show how such problem can be solved in a straightforward way using B/LMIs. In section $\mathrm{V}$ we illustrate a concrete example of stabilization of the interconnection of a DC-DC converter and a load with negative impedance characteristics. Finally, section VI contains an exposition of directions of current and future research.

\section{NOTATION}

The space of $n$ dimensional real (complex) vectors is denoted by $\mathbb{R}^{n}$ (respectively $\mathbb{C}^{n}$ ), and that of $m \times n$ real matrices by $\mathbb{R}^{m \times n}$. $\mathbb{R}^{\bullet \times m}$ denotes the space of real matrices with $m$ columns and an unspecified finite number of rows. Given matrices $A, B \in \mathbb{R}^{\bullet \times m}, \operatorname{col}(A, B)$ denotes the matrix obtained by stacking $A$ over $B$.

The ring of polynomials with real coefficients in the indeterminate $s$ is denoted by $\mathbb{R}[s]$; the ring of two-variable polynomials with real coefficients in the indeterminates $\zeta$ and $\eta$ is denoted by $\mathbb{R}[\zeta, \eta]$. $\mathbb{R}^{r \times q}[s]$ denotes the set of all $r \times q$ matrices with entries in $s$, and $\mathbb{R}^{n \times m}[\zeta, \eta]$ that of $n \times m$ polynomial matrices in $\zeta$ and $\eta$. The set of rational $m \times n$ matrices in the indeterminate $s$ is denoted by $\mathbb{R}^{m \times n}(s)$.

The set of piecewise infinitely differentiable functions from $\mathbb{R}$ to $\mathbb{R}^{q}$ is denoted by $\mathfrak{C}_{p}^{\infty}\left(\mathbb{R}, \mathbb{R}^{q}\right) . \mathfrak{D}_{p}\left(\mathbb{R}, \mathbb{R}^{q}\right)$ is the subset of $\mathfrak{C}_{p}^{\infty}\left(\mathbb{R}, \mathbb{R}^{q}\right)$ consisting of compact support functions. For a function $f:[t-\epsilon, t) \rightarrow \mathbb{R}^{\bullet}$ we set the notation $f\left(t^{-}\right):=\lim _{\tau}{ }_{t} f(\tau)$; and similarly for $f:(t, t+\epsilon] \rightarrow \mathbb{R}^{\bullet}$ we set $f\left(t^{+}\right):=\lim _{\tau \searrow t} f(\tau)$, provided that these limits exist.

\section{BACKGROUND MATERIAL}

In this paper we use standard concepts and notation of the behavioral setting, in particular those of linear differential behaviours, state maps and quadratic differential forms. A simplified collection of the theory that is relevant for the presented results can be found in App. A, p. 2046 of [9]. We now recall the basic definitions of SLDS in [9].

\section{A. Switched linear differential systems}

In our framework, each dynamical mode of a switched system is associated with a mode behavior, i.e. the set of trajectories that satisfy the dynamical laws of that particular 
mode. A switching signal determines when a transition between dynamical modes occurs. At the switching instants the system trajectories must satisfy certain gluing conditions, that represent algebraic constraints enforced by physical principles (e.g. conservation of charge/flux, kinematic constraints at interconnection). This framework has several advantages over the classical one based on state-space representations of the modes, especially in the modelling of power systems, see [10].

Definition 1: A switched linear differential system (SLDS) $\Sigma$ is a quadruple $\Sigma=\{\mathcal{P}, \mathcal{F}, \mathcal{S}, \mathcal{G}\}$ where

- $\mathcal{P}=\{1, \ldots, N\} \subset \mathbb{N}$, is the set of indices;

- $\mathcal{F}=\left\{\mathfrak{B}_{1}, \ldots, \mathfrak{B}_{N}\right\}$, with $\mathfrak{B}_{j}$ a linear differential behaviour and $j \in \mathcal{P}$, is the bank of behaviours;

- $\mathcal{S}=\{s: \mathbb{R} \rightarrow \mathcal{P}\}$, with $s$ piecewise constant and rightcontinuous, is the set of admissible switching signals; and

- $\mathcal{G}=\left\{\left(G_{k \rightarrow \ell}^{-}(s), G_{k \rightarrow \ell}^{+}(s)\right) \in \mathbb{R}^{\bullet \times \mathrm{w}}[s] \times \mathbb{R}^{\bullet \times \mathrm{w}}[s] \mid 1 \leq\right.$ $k, \ell \leq N, k \neq \ell\}$, is the set of gluing conditions.

The set of switching instants associated with $s \in \mathcal{S}$ is defined by $\mathbb{T}_{s}:=\left\{t \in \mathbb{R} \mid s\left(t^{-}\right) \neq s\left(t^{+}\right)\right\}=\left\{t_{1}, t_{2}, \ldots\right\}$, where $t_{i}<t_{i+1}$.

The set of all admissible trajectories satisfying the laws of the mode behaviours and the gluing conditions is the switched behaviour, and is the central object of study in our framework.

Definition 2: Let $\Sigma=\{\mathcal{P}, \mathcal{F}, \mathcal{S}, \mathcal{G}\}$ be a SLDS, and let $s \in \mathcal{S}$. The $s$-switched linear differential behaviour $\mathfrak{B}^{s}$ is the set of trajectories $w: \mathbb{R} \rightarrow \mathbb{R}^{\mathrm{w}}$ that satisfy the following two conditions:

1) for all $t_{i}, t_{i+1} \in \mathbb{T}_{s},\left.\left.w\right|_{\left[t_{i}, t_{i+1}\right)} \in \mathfrak{B}_{s\left(t_{i}\right)}\right|_{\left[t_{i}, t_{i+1}\right)}$;

2) $w$ satisfies the gluing conditions $\mathcal{G}$ at the switching instants for each $t_{i} \in \mathbb{T}_{s}$, i.e.

$$
\begin{aligned}
G_{s\left(t_{i-1}\right) \rightarrow s\left(t_{i}\right)}^{+} & \left(\frac{d}{d t}\right) w\left(t_{i}^{+}\right) \\
& =G_{s\left(t_{i-1}\right) \rightarrow s\left(t_{i}\right)}^{-}\left(\frac{d}{d t}\right) w\left(t_{i}^{-}\right) .
\end{aligned}
$$

The switched linear differential behaviour (SLDB) $\mathfrak{B}^{\Sigma}$ of $\Sigma$ is defined by $\mathfrak{B}^{\Sigma}:=\bigcup_{s \in \mathcal{S}} \mathfrak{B}^{s}$.

It is straightforward to verify that each trajectory in $\mathfrak{B}^{\Sigma}$ is piecewise infinitely differentiable, i.e. smooth when a mode is active and possibly discontinuous at the switching instants.

To be physically meaningful, gluing conditions should be well-defined and well-posed. In the following definition we use the notion of state maps, see [16], [19].

Definition 3: Let $\Sigma$ be a SLDS and let $X_{j} \in \mathbb{R}^{\mathrm{n}\left(\mathfrak{B}_{j}\right) \times 1}[s]$, induce minimal state maps for $\mathfrak{B}_{j}:=\operatorname{im} M_{j}\left(\frac{d}{d t}\right), j=$ $1, \ldots, N$. The gluing conditions are well-defined if there exist constant matrices $F_{j \rightarrow k}^{-}$and $F_{j \rightarrow k}^{+}$, with $j, k=1, \ldots, N$, $j \neq k$, such that $\bar{G}_{j \rightarrow k}^{-}(s)=F_{j \rightarrow k}^{-} X_{j}(s)$ and $\bar{G}_{j \rightarrow k}^{+}(s)=$ $F_{j \rightarrow k}^{+} X_{k}(s)$, with $j, k=1, \ldots, N, j \neq k$.

If $\mathcal{G}:=\left\{\left(F_{j \rightarrow k}^{-} X_{j}(s), F_{j \rightarrow k}^{+} X_{k}(s)\right)\right\}_{j, k=1, \ldots, N, j \neq k \text {. are }}$ well-defined, we call them well-posed if for all $k, j=$ $1, \ldots, N$ with $k \neq j$, there exists a re-initialisation map
$L_{j \rightarrow k}: \mathbb{R}^{\mathrm{n}\left(\mathfrak{B}_{j}\right)} \rightarrow \mathbb{R}^{\mathrm{n}\left(\mathfrak{B}_{k}\right)}$ such that given a switching signal $s \in \mathcal{S}$ such that $s\left(t_{i-1}\right)=j$ and $s\left(t_{i}\right)=k$; for all $t_{i} \in \mathbb{T}_{s}$ and all admissible $w \in \mathfrak{B}^{\Sigma}$ with associated latent variable trajectories, it holds that $X_{j}\left(\frac{d}{d t}\right) \ell_{j}\left(t_{i}^{+}\right)=$ $L_{k \rightarrow j} X_{k}\left(\frac{d}{d t}\right) \ell_{k}\left(t_{i}^{-}\right)$.

\section{B. Passive switched linear differential systems}

In the following we consider SLDS with controllable modes having $m$ inputs and $m$ output variables; it follows that every mode can be described in image form (see [15])

$$
w=M_{i}\left(\frac{d}{d t}\right) \ell_{i},
$$

where $\ell_{i} \in \mathfrak{D}_{p}\left(\mathbb{R}, \mathbb{R}^{m}\right), i=1, \ldots, N$; and $M_{i} \in \mathbb{R}^{q \times m}[s]$ is such that $M_{i}=\operatorname{col}\left(U_{i}, Y_{i}\right)$ with $U_{i}, Y_{i} \in \mathbb{R}^{m \times m}[s]$ and $U_{i}$ nonsingular, $i=1, \ldots, N$.

To define passive SLDS, we first introduce the following notation. Let $s \in \mathcal{S}$ be a fixed switching signal, with associated set of switching instants $\mathbb{T}_{s}:=\left\{t_{1}, t_{2}, \ldots, t_{n}, \ldots\right\}$. We denote by $\left|\mathbb{T}_{s}\right|$ the total number of switching instants in $\mathbb{T}_{s}$. Let

$$
\Phi:=\frac{1}{2}\left[\begin{array}{cc}
0_{m \times m} & I_{m} \\
I_{m} & 0_{m \times m}
\end{array}\right] \in \mathbb{R}^{q \times q},
$$

and let $w \in \mathfrak{B}^{\Sigma}$.

If $\left|\mathbb{T}_{s}\right|=\infty$, define $\int Q_{\Phi}(w):=\int_{-\infty}^{t_{1}^{-}} Q_{\Phi}(w) d t+$ $\int_{t_{1}^{+}}^{t_{2}^{-}} Q_{\Phi}(w) d t+\ldots+\int_{t_{n}^{+}}^{t_{n+1}^{-}} Q_{\Phi}(w) d t+\ldots$.

If $0<\left|\mathbb{T}_{s}\right|<\infty$, then define $\int Q_{\Phi}(w):=$ $\int_{-\infty}^{t_{1}^{-}} Q_{\Phi}(w) d t+\sum_{k=2}^{\left|\mathbb{T}_{s}\right|} \int_{t_{k-1}^{+}}^{t_{k}^{-}} Q_{\Phi}(w) d t+\int_{t_{\left|\mathbb{T}_{s}\right|}^{+}}^{\infty} Q_{\Phi}(w) d t$

If $\left|\mathbb{T}_{s}\right|=0$, i.e. no switching takes place, then $\int Q_{\Phi}(w):=\int_{-\infty}^{+\infty} Q_{\Phi}(w) d t$.

The definition of passive SLDS is as follows.

Definition 4: Let $\Sigma$ be a SLDS and define $\Phi$ as in (2). $\Sigma$ is passive if $\int Q_{\Phi}(w) \geq 0$ for all $w \in \mathfrak{B}^{\Sigma} \cap \mathfrak{D}_{p}\left(\mathbb{R}, \mathbb{R}^{\mathrm{w}}\right)$.

Sufficient conditions for the passivity of a given SLDS can be given in terms of LMIs, and they are instrumental in our solution to the constant power load problem; we will review them later on in this paper (see Prop. 1 below).

\section{A SWITCHED LINEAR DIFFERENTIAL SYSTEM MODEL FOR THE CONSTANT POWER LOAD PROBLEM}

We formalize the constant power load problem in the SLDS framework as follows. We are given a switched linear differential system (corresponding to the box labeled $\Sigma$ in Fig. 1) whose dynamical modes (corresponding to the regimes induced in a single power converter by an ideal switch) are described as two-ports. As in [3], the destibilizing effects of the constant power load are locally approximated using a negative impedance load (the box labelled CPL in Fig. 1) and the passive filter (represented by a parameterdependent impedance with a priori fixed McMillan degree $n$ ) are described by one-port.

Proposition 1: Assume that the dynamical modes of the switching power converter $\Sigma$ in Fig. 1 can be described in image form $w=M_{j}\left(\frac{d}{d t}\right) \ell_{j}$, with $M_{j} \in \mathbb{R}^{4 \times 2}[s]$, 


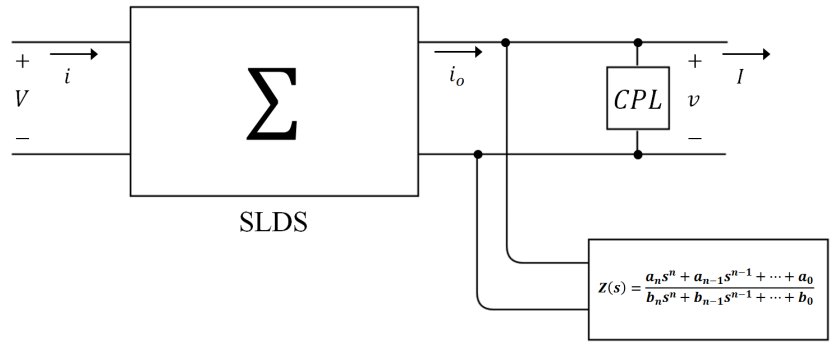

Fig. 1. Distributed power system with passive damping.

$j=1, \ldots, N ; \ell_{j}=\operatorname{col}\left(\ell_{1, j}, \ell_{2, j}\right) \in \mathfrak{D}_{p}\left(\mathbb{R}, \mathbb{R}^{2}\right), i=1, \ldots N ;$ and $w:=\left[\begin{array}{llll}V & i_{o} & i & v\end{array}\right]^{\top}$.

Let $\ell_{f} \in \mathfrak{D}_{p}(\mathbb{R}, \mathbb{R})$, then there exist $\widehat{M}_{k} \in \mathbb{R}^{4 \times 2}[s]$ such that the $k$-th dynamical regime of the interconnection in Fig. 1 is described by an image representation

$$
\left[\begin{array}{c}
V \\
I \\
i \\
v
\end{array}\right]=\widehat{M}_{k}\left(\frac{d}{d t}\right)\left[\begin{array}{c}
\ell_{1, k} \\
\ell_{f}
\end{array}\right],
$$

$k=1, \ldots, N$. Moreover, denote by $\frac{q(s)}{p(s)}$ the impedance of the filter; then each entry of $\widehat{M}_{k}(s)$ depends at most linearly on $q(s)$ and $p(s)$, i.e. $\widehat{M}_{k}(s)=\widehat{M}_{k}^{1}(s)+\widehat{M}_{k}^{2}(s) q(s)+$ $\widehat{M}_{k}^{3}(s) p(s)$, where $M_{k}^{i} \in \mathbb{R}^{4 \times 2}[s], k=1, \ldots, N, i=1,2,3$.

Proof: The negative impedance load (CPL) generating instability is described by a one-port, and consequently can also be represented in observable image representation by $M^{\prime} \in \mathbb{R}^{2 \times 1}[s]$ with external variables $w^{\prime}:=\left[\begin{array}{ll}I^{\prime \prime} & v\end{array}\right]^{\top}$ and one-dimensional latent variable denoted by $\ell^{\prime}$.

The passive filter is a one-port impedance that can be represented by an observable image representation $M_{f} \in$ $\mathbb{R}^{2 \times 1}[s]$ with external variable $w_{f}:=\left[\begin{array}{ll}I^{\prime} & v\end{array}\right]^{\top}$ and onedimensional latent variable denoted by $\ell_{f}$.

It follows from the elimination theorem (see Sec. 6 of [15]) that the interconnection of the negative impedance load and the filter has a representation as a one-port, with latent variable $\ell_{f}$. After elimination of the latent variable $\ell_{2, k}$ for each mode, the interconnection of this one-port impedance with the plant has dynamical modes that can be described as two-ports, corresponding to the image representations (3).

The parameters of such image representation depend only linearly on the filter parameters since elimination of the latent variable $\ell_{2, k}$ is performed by computing the syzygy of the module generated by a polynomial column vector independent of $q(s)$ and $p(s)$.

The following is an important consequence of Prop. 1.

Corollary 1: The state maps for the SLDS associated with the behaviors described in image form by (3) depend linearly on the coefficients of the polynomials $q(s)$ and $p(s)$ describing the filter.

Proof: A state map can be computed from an image representation via algebraic operations as follows (see [16], [19]). Let $\widehat{U}_{k} \in \mathbb{R}^{2 \times 2}[s]$ be a maximal determinantal degree submatrix of $\widehat{M}_{k}, k=1, \ldots, N$. Define $X_{k}^{1}(s):=$
$s^{-1}\left(U_{k}(s)-U_{k}(0)\right), X_{k}^{2}(s):=s^{-1}\left(X_{k}^{1}(s)-X_{k}(0)\right)$, and so forth. Then the polynomial differential operator induced by the matrix $X_{k}(s):=\operatorname{col}\left(X_{k}^{i}\right)_{i=1, \ldots}$ and acting on the latent variable $\ell_{k}$ induces a state variable. Each entry of such polynomial matrix depend linearly on the coefficients of $q(s)$ and $p(s)$, since $\widehat{U}_{k}(s)$ itself does. This concludes the proof.

We now prove that under mild assumptions satisfied for all reasonable electric circuits, the gluing conditions of the switched linear differential system corresponding to Fig. 1 are well-defined according to Def. 3.

Proposition 2: Assume that switching among the modes described by (3) does not involve short-circuiting of voltage sources, or open-circuiting of current sources. Then the gluing conditions are well-defined.

Proof: If switching between modes does not involve short- or open-circuiting sources, no constraints on the input variables of the system are imposed at the switching instants. Consequently, the gluing conditions only impose constraints on the output variables of the modes, which are linear functions of the state variables. The claim follows.

Well-posed gluing conditions (see Def. 3) guarantee that after a switching instant only one initial state for the new dynamical regime is specified from the final state of the previous one. Such property holds since the switching cannot cause any increase in the total amount of charge or flux stored in the system and thus the value of the state after the switch is uniquely determined by the state before the switch. On this issue, see [13] where the analysis of a wide variety of physical systems exhibiting discontinuities is presented, and [4], [5], [18]. In the rest of this paper we assume that the gluing conditions are well-posed.

\section{A DESIGN METHODOLOGY FOR CONSTANT-POWER LOAD STABILIZATION BY PASSIVE DAMPING}

The idea underlying our methodology is to connect a passive filter between the constant power load and the SLDS, so that the overall system is passive and consequently $\mathrm{i} / \mathrm{o}$ stable (see Th. 6 of [9]). Our methodology for finding such a filter is based on the following result (see Th. 4 of [9]) providing an LMI-based test for passivity of SLDS.

In the following, the coefficient matrix of $F(s)=$ $\sum_{i=0}^{N} F_{i} s^{i} \in \mathbb{R}^{q_{1} \times q_{2}}[s]$ is defined by

$$
\widetilde{F}:=\left[\begin{array}{llll}
F_{0} & F_{1} & \ldots & F_{N}
\end{array}\right] .
$$

Note that $F(s)=\widetilde{F}\left[\begin{array}{llll}I_{q_{2}} & s I_{q_{2}} & \ldots & I_{q_{2}} s^{N}\end{array}\right]^{\top}$.

Theorem 1: Let $\Sigma$ be a SLDS with $\mathcal{G}$ well-defined and well-posed. Let $X_{k} \in \mathbb{R}^{\mathrm{n}\left(\mathfrak{B}_{k}\right) \times 1}[s]$ be a minimal state map for $\mathfrak{B}_{k}, i=1, \ldots, N$, and let $L_{i \rightarrow j} \in \mathbb{R}^{\mathrm{n}\left(\mathfrak{B}_{j}\right) \times \mathrm{n}\left(\mathfrak{B}_{i}\right)}$ for all $i, j \in \mathcal{P}, i \neq j$, be the re-initialisations maps of $\Sigma$. Denote the coefficient matrix of $M_{k}(s)$ by $\widetilde{M}_{k}:=$ $\left[\begin{array}{lll}M_{k, 0} & \ldots & M_{k, L_{k}}\end{array}\right]$; then that of $X_{k}(s)$ can be written as $\widetilde{X}_{k}:=\left[\begin{array}{lll}X_{k, 0} & \ldots & X_{k, L_{k}-1}\end{array}\right]$.

If there exist $K_{k}=K_{k}^{\top} \in \mathbb{R}^{\mathrm{n}\left(\mathfrak{B}_{k}\right) \times \mathrm{n}\left(\mathfrak{B}_{k}\right)}, k=1 \ldots, N$, 
such that

$$
\begin{aligned}
& \widetilde{M}_{k}^{\top} \Phi \widetilde{M}_{k}-\left[\begin{array}{c}
0_{1 \times \mathrm{n}\left(\mathfrak{B}_{k}\right)} \\
\widetilde{X}_{k}^{\top}
\end{array}\right] K_{k}\left[\begin{array}{ll}
\widetilde{X}_{k} & 0_{\mathrm{n}\left(\mathfrak{B}_{k}\right) \times 1}
\end{array}\right] \\
& -\left[\begin{array}{c}
\widetilde{X}_{k}^{\top} \\
0_{1 \times \mathrm{n}\left(\mathfrak{B}_{k}\right)}^{\top}
\end{array}\right] K_{k}\left[\begin{array}{ll}
0_{\mathrm{n}\left(\mathfrak{B}_{k}\right) \times 1} & \widetilde{X}_{k}
\end{array}\right] \geq 0,
\end{aligned}
$$

and moreover, if for $k, j=1, \ldots, N, k \neq j$, it holds that

$$
K_{k}-L_{k \rightarrow j}^{\top} K_{j} L_{k \rightarrow j} \geq 0,
$$

then $\Sigma$ is passive.

In the result of Th. 1, the LMIs (4) correspond to the (necessary) conditions on the passivity of each mode, and the LMIs (5) to the (sufficient) conditions that the stored energy does not increase at the switching instants, see [9].

The first step in our procedure is to obtain from the switching power converter, a negative impedance load ( e.g. as in [3]), and a filter parametric description $\frac{q(s)}{p(s)}$, image representations $\widehat{M}_{k}$ as in Prop. 1, and the corresponding gluing conditions. It follows from Prop. 1, Cor. 1 and Prop. 2 that the polynomial matrices $\widehat{M}_{k}, G_{k \rightarrow \ell}^{-}, G_{k \rightarrow \ell}^{+}$and the state maps $X_{k}$ depend linearly on the unknown parameters corresponding to the coefficients of the passive filter $\frac{q(s)}{p(s)}$.

The second step in our procedure is the setting up of a system of matrix inequalities corresponding to the conditions of Th. 1. To make explicit the linear dependence on the filter parameters, in the following we write $\widehat{M}_{k}(s), X_{k}(s)$ respectively as $\widehat{M}_{k, \widetilde{p}, \widetilde{q}}(s)$ and $X_{k, \widetilde{p}, \widetilde{q}}(s)$, where $\widetilde{p}, \widetilde{q}$ are the coefficient matrices of the unknown denominator and numerator of the passive filter:

$$
\begin{aligned}
& \widetilde{M}_{k, \widetilde{p}, \widetilde{q}}^{\top} \Phi \widetilde{M}_{k, \widetilde{p}, \widetilde{q}}-\left[\begin{array}{c}
0_{1 \times \mathrm{n}\left(\mathfrak{B}_{k}\right)} \\
\widetilde{X}_{k, \widetilde{p}, \widetilde{q}}^{\top}
\end{array}\right] K_{k}\left[\begin{array}{ll}
\widetilde{X}_{k, \widetilde{p}, \widetilde{q}} & 0_{\mathrm{n}\left(\mathfrak{B}_{k}\right) \times 1}
\end{array}\right] \\
& -\left[\begin{array}{c}
\widetilde{X}_{k, \widetilde{p}, \widetilde{q}}^{\top} \\
0_{1 \times \mathrm{n}\left(\mathfrak{B}_{k}\right)}
\end{array}\right] K_{k}\left[\begin{array}{ll}
0_{\mathrm{n}\left(\mathfrak{B}_{k}\right) \times 1} & \widetilde{X}_{k, \widetilde{p}, \widetilde{q}}
\end{array}\right] \geq 0, \\
& k=1, \ldots, N \\
& K_{k}-L_{k \rightarrow j}^{\top} K_{j} L_{k \rightarrow j} \geq 0, k, j=1, \ldots, N, k \neq j .(6
\end{aligned}
$$

The third step is to formalize the requirement that the filter is passive. Define

$$
\begin{aligned}
\Phi^{\prime} & :=\frac{1}{2}\left[\begin{array}{ll}
0 & 1 \\
1 & 0
\end{array}\right], \\
M^{\prime}(s) & :=\left[\begin{array}{c}
p(s) \\
q(s)
\end{array}\right] \\
X^{\prime}(s) & :=\left[\begin{array}{c}
1 \\
s \\
\vdots \\
s^{\operatorname{deg}(p)-1}
\end{array}\right],
\end{aligned}
$$

and denote the coefficient matrices of $M^{\prime}$ and $X^{\prime}$ by $\widetilde{M}^{\prime} \widetilde{p}, \widetilde{q}$ and $\widetilde{X^{\prime}}$, respectively. With these positions, it follows from the positive-real lemma that $\frac{q}{p}$ is positive-real if and only if there exists $K^{\prime}=K^{\prime \top} \in \mathbb{R}^{\operatorname{deg}(p) \times \operatorname{deg}(p)}$ such that

$$
\begin{aligned}
& \widetilde{M}_{\widetilde{p}, \widetilde{q}}^{\prime \top} \Phi^{\prime} \widetilde{M}_{\widetilde{p}, \widetilde{q}}^{\prime}-\left[\begin{array}{c}
0_{1 \times \operatorname{deg}(p)}^{\widetilde{X}^{\prime \top}}
\end{array}\right] K^{\prime}\left[\begin{array}{ll}
\widetilde{X}^{\prime} & 0_{\operatorname{deg}(p) \times 1}
\end{array}\right] \\
& -\left[\begin{array}{c}
\widetilde{X}^{\prime \top} \\
0_{1 \times \operatorname{deg}(p)}
\end{array}\right] K^{\prime}\left[\begin{array}{ll}
0_{\operatorname{deg}(p) \times 1} & \widetilde{X}^{\prime}
\end{array}\right] \geq 0 .
\end{aligned}
$$

If values of the parameters $\widetilde{p}$ and $\widetilde{q}$ exist such that the matrix inequalities (6),(8) are satisfied for some $K_{k}$, $k=1, \ldots, N$ and $K^{\prime}$, then the interconnection of Fig. 1 is passive, and consequently i/o stable. Moreover, the filter $\frac{q}{p}$ can be implemented using only resistors, capacitors, inductors and transformers (see [14]).

In the following remarks we discuss several important issues about our procedure to solve the constant load problem by passive damping.

Remark 1: The McMillan degree $n$ of the stabilizing passive filter (assuming it exists) is not known a priori. To start our procedure, some value of $n$ should be decided upon, and a solution attempted to the matrix inequalities (6),(8). If no solution exists for the current value of $n$, the latter should be increased, and the procedure repeated.

It is a matter for future research to establish upper bounds on the McMillan degree of the filter in terms of the complexity of the plant dynamical modes and of that of the destabilizing negative impedance, assuming such a filter exists.

Remark 2: The inequalities (6) and (8) are bilinear in the coefficients of the polynomials $p$ and $q$ of the passive filter. In some special cases they can be made linear by fixing the values of certain parameters, for example by deciding a priori what the poles/zeros of the filter should be. See section V of this paper for an instance of this approach.

Remark 3: A common assumption in the known approaches to the constant load problem is to model the destabilizing load as a negative resistance (see e.g. [1]). In our methodology we do not restrict the class of load models; for example we admit frequency-domain models of as differences of squares $\left(P(-s)^{\top} P(s)-N(-s)^{\top} N(s)\right.$ ). This may lead to less conservative solutions of the constant load problem, for example leading to the design of filters that induce a smaller power loss than those arrived at by conventional means. This is a matter for future research.

\section{EXAMPLE}

Consider the DC-DC power converter in Fig. 2 which is interconnected to a constant power load whose destabilizing effects are represented by the impedance $Z_{C P L}$ (see [3]). As explained in Sec. IV, we introduce a passive filter (a circuit consisting of inductors, capacitors and resistors) described by an admittance $Y$ that when connected to the switched network, the overall circuit is passive.

The filter is a 1-port impedance function $Z(s)=\frac{q(s)}{p(s)}$, with $p, q \in \mathbb{R}[s]$, or equivalently an image representation

$$
\left[\begin{array}{c}
v \\
i_{f}
\end{array}\right]=\underbrace{\left[\begin{array}{l}
p\left(\frac{d}{d t}\right) \\
q\left(\frac{d}{d t}\right)
\end{array}\right]}_{=: M^{\prime}\left(\frac{d}{d t}\right)},
$$




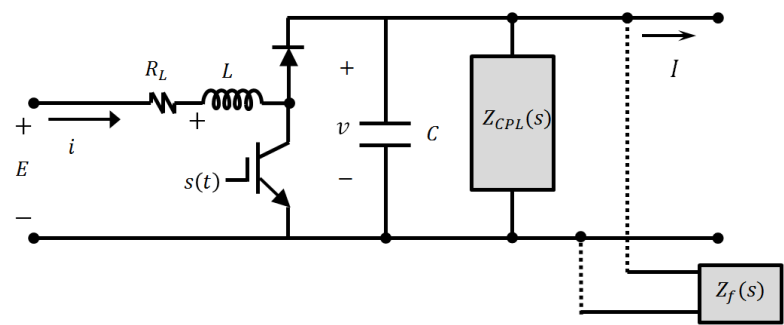

Fig. 2. DC-DC converter with a constant power load and a stabilizing filter.

where $\ell^{\prime}$ is a latent variable associated to the internal dynamics of the filter and whose physical meaning can be determined after the realisation. Similarly, the dynamics of the negative impedance are $Z_{C P L}(s)=-R$. Applying fundamental current and voltage laws, the mode dynamics when the transistor is closed and the diode open are described by

$$
\begin{aligned}
V & =L \frac{d}{d t} i_{1}+R_{L} i_{1}, \\
I & =C \frac{d}{d t} v-\frac{1}{R} v+q\left(\frac{d}{d t}\right) \ell^{\prime} .
\end{aligned}
$$

When the transistor is open and the diode is closed, the mode dynamics are described by

$$
\begin{aligned}
V & =L \frac{d}{d t} i_{1}+R_{L} i_{1}+v \\
I & =-i_{1}+C \frac{d}{d t} v-\frac{1}{R} v+q\left(\frac{d}{d t}\right) \ell^{\prime} .
\end{aligned}
$$

By selecting the external and latent variables as $w:=$ $\left[\begin{array}{llll}V & I & i_{1} & v\end{array}\right]^{\top}$ and $\ell:=\left[\begin{array}{ll}i_{1} & \ell^{\prime}\end{array}\right]^{\top}$, the mode dynamics can be modelled using image form representations $w=$ $M_{k}\left(\frac{d}{d t}\right) \ell, k=1,2$, where

$$
\begin{aligned}
M_{1}(s) & :=\left[\begin{array}{cc}
L s+R_{L} & 0 \\
0 & -\left(\frac{1}{R}-C s\right) p(s)+q(s) \\
1 & 0 \\
0 & p(s)
\end{array}\right] \\
M_{2}(s) & :=\left[\begin{array}{cc}
L s+R_{L} & p(s) \\
-1 & -\left(\frac{1}{R}-C s\right) p(s)+q(s) \\
1 & 0 \\
0 & p(s)
\end{array}\right] .
\end{aligned}
$$

Note that as described in Prop. 1, the dependence of $M_{1}$ and $M_{2}$ on the unknown parameters of the filter is linear.

State maps for $\mathfrak{B}_{i}, i=1,2$ and the filter can be computed e.g. as

$$
\begin{aligned}
X_{1}(s) & :=\left[\begin{array}{cccc}
1 & 0 & 0 & 0 \\
0 & 1 & s & s^{2}
\end{array}\right]^{\top}=: X_{2}(s), \\
X^{\prime}(s) & :=\left[\begin{array}{ll}
1 & s
\end{array}\right]^{\top} .
\end{aligned}
$$

In this particular case the matrices $\widetilde{X}_{i}, i=1,2$ (the coefficient matrices of state maps for the dynamical modes) and the coefficient matrices $\widetilde{M}_{i}, i=1,2$, do not depend on the unknown coefficients associated to the filter (the general case is covered in Prop. 1 and Cor. 1). It follows from the physics of the switched circuit that the re-initialisation maps are $L_{1 \rightarrow 2}=L_{2 \rightarrow 1}=I_{4}$.

It is straightforward to see that the matrix inequalities (6) and (8) associated with the models (9) and the choice of the state maps (10) are bilinear in the coefficients of $p(s)$ and $q(s)$. Given the particular structure of our problem, if we fix the coefficients of the polynomial $p$, linear matrix inequalities occur, depending only on the coefficients of $q(s)$. Fixing $p$ is tantamount to fixing the poles of the filter; this is often a reasonable choice, since filter performance specifications such as time response, characteristic frequencies etc., are directly related to the position of the poles of the filter in the complex plane (see Sec. 5-8 of [17]).

Now assume that $R_{L}=0.01 \Omega, L=500 \mu \mathrm{H}, C=47 \mu \mathrm{F}$ and $-R=-300 \Omega$. Define $p(s):=7392000+87200 s+$ $160 s^{2}$, and let $p(s):=a_{2} s^{2}+a_{1} s+a_{0}$. The selected purely real roots of $q$ correspond to the realisation of an $R C$ lowpass circuit (see [17], Sec. 3).

Using standard LMI solvers for such choice of $q$, the coefficients of the polynomial $p$ resulting from the solution of the LMIs, yield $p(s)=14000+270 s+s^{2}$. Consequently the filter described by the impedance

$$
Z(s)=\frac{7392000+87200 s+160 s^{2}}{14000+270 s+s^{2}}
$$

stabilizes the interconnection. Note that such filter can be physically realised using any suitable method of passive circuit synthesis (see [14]); for example, the circuit in Fig. 3 represents a Cauer realization of $Z(s)$, with $R_{1}=160 \Omega$, $R_{2}=287.75 \Omega, R_{3}=80.24 \Omega, C_{1}=22.72 \mu F$ and $C_{1}=$ $136.105 \mu \mathrm{F}$

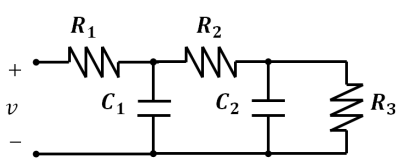

Fig. 3. Filter realisation.

We now show simulation results of the circuit in Fig. 2, when the stabilizing filter is connected. In order to do so we set the input voltage $E=50$ volts and initial conditions equal to zero. The switching signal used for the simulation is periodical, and corresponds to a traditional PWM (pulse width modulation), i.e.

$$
s(t)= \begin{cases}1, & t_{k} \leq t<t_{k}+D T \\ 2, & t_{k}+D T \leq t<t_{k}+T .\end{cases}
$$

where $D=0.5, T=50 \mu s, t_{0}:=0$ and $t_{k+1}=t_{k}+T$, with $k=0,1,2, \ldots$.

The simulation was carried out using Synopsys Saber and the results are shown in Fig. 4 and Fig. 5, which depict the transients of the voltage across the capacitor and the current through the inductor of the converter. We compare the cases when the stabilising filter is respectively disconnected 
and connected. Note that the stabilization effect of the filter corroborates the theoretical principles that are used to support the proposed method.

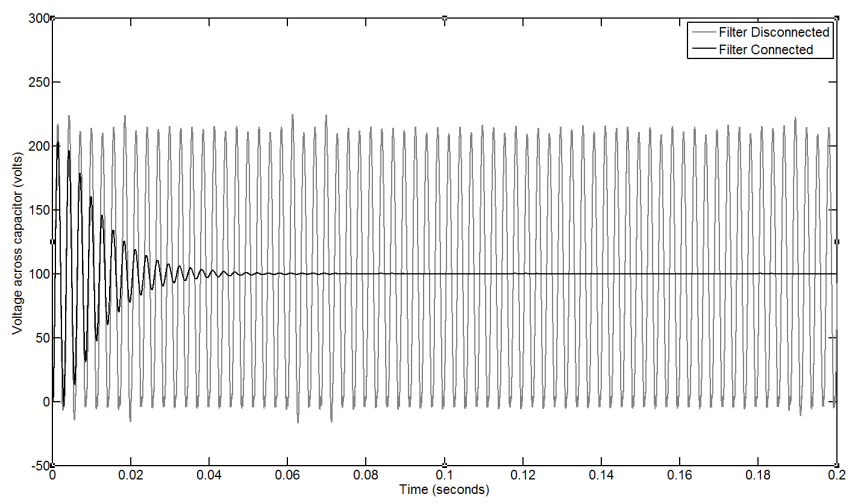

Fig. 4. Comparison between simulations of the output voltage of the converter when the filter is disconnected and connected.

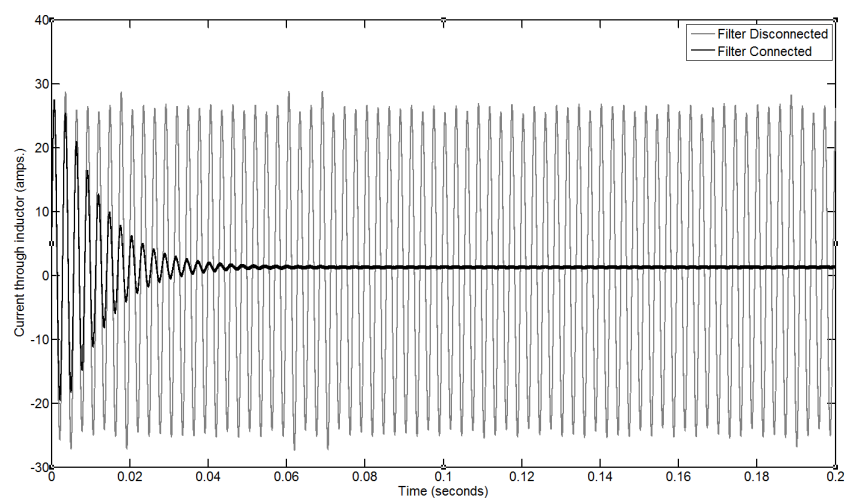

Fig. 5. Comparison between simulations of the input current of the converter when the filter is disconnected and connected.

\section{CONCLUSIONS}

We have illustrated a systematic design procedure for the solution of the constant power load problem. This procedure is founded on the passivation of the combination of the plant and the instability-generating negative impedance by interconnecting to it a suitable passive filter. The switched linear differential framework, based as it is on image representations, accommodates in a natural way impedance representations of the plant, filter, and negative impedance, so that the formulation of the problem in terms of the solution to a system of bilinear matrix inequalities is straightforward. In case some design parameters are known or fixed a priori, the matrix inequalities may turn out to be linear, and thus more easily tractable by standard LMI solvers. Our methodology has been demonstrated with an example of a DC-DC converter connected to a negative resistor.

\section{REFERENCES}

[1] M. Cespedes, Lei Xing, and Jian Sun. Constant-power load system stabilization by passive damping. Power Electronics, IEEE Transactions on, 26(7):1832-1836, July 2011.
[2] W. Du, J. Zhang, Y. Zhang, and Z. Qian. Stability criterion for cascaded system with constant power load. IEEE Trans. Power Electr., 28(4):1843-1851, 2013.

[3] A. Emadi, A. Khaligh, C.H. Rivetta, and G.A. Williamson. Constant power loads and negative impedance instability in automotive systems: definition, modeling, stability, and control of power electronic converters and motor drives. IEEE Trans. Veh. Technol., 55(4):1112-1125, July 2006.

[4] R. Frasca, M.K. Camlibel, I.C. Goknar, L. Iannelli, and F. Vasca. Linear passive networks with ideal switches: Consistent initial conditions and state discontinuities. IEEE Trans. Circuits Syst. I, Reg. Papers, 57(12):3138-3151, 2010

[5] I. Goknar. Conservation of energy at initial time for passive RLCM network. IEEE Trans. Circuit Theory, 19(4):365-367, 1972.

[6] H. Lin and P.J. Antsaklis. Stability and stabilizability of switched linear systems: A survey of recent results. IEEE Trans. Aut. Contr., 54(2):308-322, 2009.

[7] J. Löfberg. Yalmip : A toolbox for modeling and optimization in MATLAB. In Proceedings of the CACSD Conference, Taipei, Taiwan, 2004.

[8] J.C. Mayo-Maldonado and P. Rapisarda. On positive-realness and stability of switched linear differential systems. Proc. 53nd IEEE CDC, 2013.

[9] J.C. Mayo-Maldonado and P. Rapisarda. Dissipative switched linear differential systems. IEEE Trans. Aut. Cont., submitted, 2014.

[10] J.C. Mayo-Maldonado and P. Rapisarda. Modelling of switching dynamics in electrical systems. Proc. Mathematical Theory of Networks and Systems Symposium (MTNS), 2014.

[11] J.C. Mayo-Maldonado, P. Rapisarda, and P. Rocha. Stability of switched linear differential systems. IEEE Trans. Aut. Cont., 59(8):2038-2051, 2014.

[12] J.C. Mayo-Maldonado, J.C. Rosas-Caro, and P. Rapisarda. Modeling approaches for DC-DC converters with switched capacitors. IEEE Trans. Ind. Electr., 62(2):953-959, Feb 2015.

[13] P.J. Mosterman and G. Biswas. A theory of discontinuities in physical system models. J. Franklin Inst., 335(3):401 - 439, 1998.

[14] R. Newcomb. Linear Multiport Synthesis. New York: McGraw-Hill, 1966.

[15] J.W. Polderman and J.C. Willems. Introduction to Mathematical System Theory: A Behavioral Approach. Springer, Berlin, 1997.

[16] P. Rapisarda and J.C. Willems. State maps for linear systems. SIAM J. Control Optim., 35(3):1053-1091, 1997.

[17] G.C. Temes and J.W. Lapatra. Introduction to circuit synthesis and design, volume 15. McGraw-Hill New York, 1977.

[18] J. Tolsa and M. Salichs. Analysis of linear networks with inconsistent initial conditions. IEEE Trans. Circuits Syst. I, Fundam. Theory, 40(12):885-894, 1993.

[19] A. van der Schaft and P. Rapisarda. State maps from integration by parts. SIAM J. Control Optim., 49(6):2415-2439, 2011.

[20] F. Xiaogang, L. Jinjun, and F.C. Lee. Impedance specifications for stable DC distributed power systems. IEEE Trans. Power Electr., 17(2):157-162, 2002.

[21] X. Zhang, X. Ruan, H. Kim, and C. K. Tse. Adaptive active capacitor converter for improving stability of cascaded dc power supply system. Power Electronics, IEEE Trans. Power Electr., 28(4):1807-1816, Apr 2013.

[22] Q.C. Zhong and T. Hornik. Control of power inverters in renewable energy and smart grid integration. John Wiley \& Sons, 2012. 